\title{
Epitaxial growth with elastic interaction: Submonolayer island formation
}

\author{
F. Gutheim, H. Müller-Krumbhaar, and E. Brener \\ Institut für Festkörperforschung, Forschungszentrum Jülich, D-52425 Jülich, Germany
}

(Received 4 October 2000; published 26 March 2001)

\begin{abstract}
A model for island formation in submonolayer epitaxy has been studied in the presence of elastic strain by means of a Monte Carlo simulation. The description, based on rate equations, leads to scaling predictions for cluster statistics and diffusion rates. We generalize these predictions to include the effects of the repulsive elastic interaction. The elastic interaction is caused by the deformation of the underlying substrate and has a repulsive $1 / r^{3}$ character. To enable the efficient simulation of multiparticle surface diffusion with long-range interaction, we employ a multigrid scheme. One particular result is that, with increasing elastic repulsion between the adsorbed particles, the formation of islands is hampered, and island nucleation is deferred to higher coverage values.
\end{abstract}

DOI: 10.1103/PhysRevE.63.041603

PACS number(s): 81.15.Aa, 68.55.-a, 81.30.Fb

\section{INTRODUCTION}

Heteroepitaxial growth is a process of great interest in crystal growth [1-3] and for the manufacturing of semiconductor devices. One has to assume that in the majority of cases elastic strain influences the properties of growth and gives rise to a variety of growth morphologies. The lattice mismatch between adsorbed layers and a substrate of different material leads to the deformation of both the adsorbate and substrate. Up to some critical size, the adsorbate will adopt the lattice structure of the substrate, apart from a local change in the lattice parameter. This "coherent" lattice deformation typically leads to a repulsive long-range interaction potential between any two adsorbed atoms, which depends on their distance $r$ like $1 / r^{3}$ [4-7] at long distances, and is mediated by the substrate. Here we will be concerned with the early stage of island nucleation in a system with a fixed deposition flux. In the low temperature limit desorption can be neglected, and adatoms are incorporated to islands irreversibly. We will first describe the model and the results from our Monte Carlo simulation, and then proceed to a scaling theory based on rate equations and some basic assumptions.

\section{MODEL AND SIMULATION RESULTS}

The system is realized on a two-dimensional $L \times L$ sized simple square lattice with a lattice parameter $\Delta x$. As we mean to model molecular beam epitaxy, we assume the deposition process to be ballistic in the sense that deposition onto the surface is not biased by the local chemical potential. The rate of deposition $F$ (measured in number of particles per $\mathrm{cm}^{2}$ and second) is equal for all lattice sites. Single adatoms, here referred to as monomers, diffuse on the surface, interacting by a repulsive potential $U_{0} / r^{3}$, which originates from the deformation of the substrate. We assume that the temperature $T$ is low enough to neglect desorption and to satisfy a critical island size of 2 ; i.e., once two monomers have met, they form a stable dimer which grows by the incorporation of further monomers. Monomers can in principle be deposited on top of existing islands, in which case they simply diffuse on top until they reach the edge of the island or form an island. Modeling elastic relaxation on higher layers, the elastic interaction decays exponentially within the scale of the mean island width when moving to higher layers of the adsorbate. For the aspects we will discuss here, the influence of higher layers is of minor importance.

Starting with zero coverage, a fixed number of particles $N=A F \Delta t$ is deposited onto a substrate of size $A=L^{2} \Delta x^{2}$ at the beginning of each time step $\Delta t$. The implementation of monomer diffusion obeys the following rules. On average, every monomer is chosen once to perform a diffusion step trial during the interval $\Delta t$. One of the four neighboring sites is chosen at random, and the move is accepted with a probability $p$ depending on $\Delta U$, the difference in elastic energy. The probability $p$ is chosen according to Metropolis rules, i.e., $p=1$ if $\Delta U / T<0$, and $p=\exp (-\Delta U / T)$ otherwise. With $\Delta x=1$ and $\Delta t=1$, the algorithm corresponds to a diffusion constant of $D=1 / 4$. In the following all length scales will be given in units of a lattice constant.

In order to efficiently evaluate the difference $\Delta U$, we employ a multigrid scheme based on Ref. [8], which avoids introducing a potential cutoff by treating the interaction with distant adsorbate atoms on a coarse grained level in the manner of a multipole expansion. The simulation was carried out using a system of size $L=1024$ with periodic boundary conditions in both spatial dimensions. The results were averaged over 4-16 runs, depending on the flux. Figures 1 and 2 show the number of monomers, $\rho_{1}$, and the number of islands, $\rho$, during the deposition process. The number of monomers increases almost linearly with the flux until it reaches its maximum. Afterward the number of monomers decreases, while the number of islands finally supersedes the number of monomers and increases until the coverage is almost one monolayer, where island coalescence leads to a rapid decrease of the number of islands. Until then contributions from higher layers can be ignored, and thus only particles from the first monolayer are taken into account for the computation of monomer and island densities. Nevertheless the model allows for multilevel island formation. With increasing ratio $D / F$ the maximum number of the monomers decreases, and is shifted to lower coverages. This refers to simulations both with and without interaction. If one compares the data for different interaction strengths $U_{0} / T$, as 


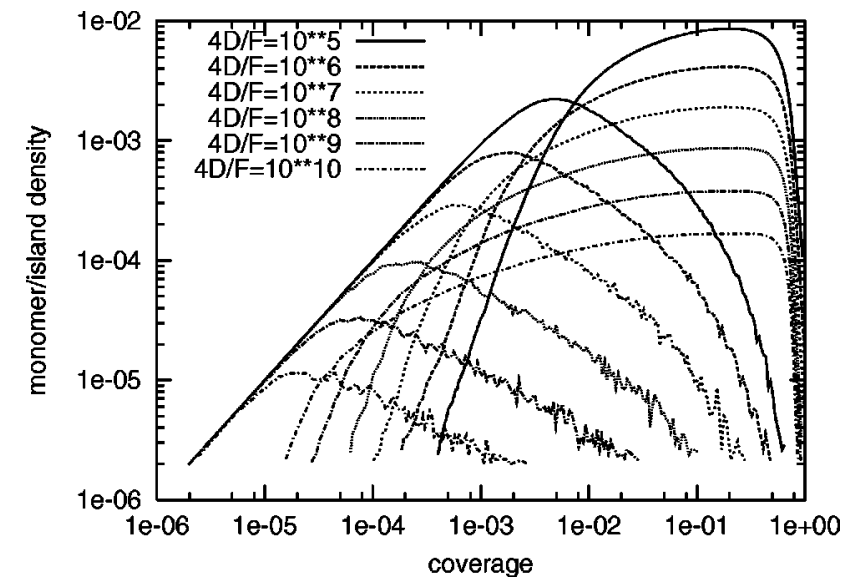

FIG. 1. Monomer $\rho_{1}$ and island densities $\rho$ vs coverage $\theta$ without elastic repulsion $U_{0} / T=0$, with $4 D / F$ ranging from $10^{5}$ to $10^{10}$.

plotted in Fig. 3 for identical $D / F$, one notes that interaction shifts the maximum of the monomer density toward higher coverage values because repulsion will drive monomers apart, hampering the formation of dimers.

\section{SCALING THEORY}

A newly deposited monomer will diffuse during a characteristic time $\tau_{c}$, until eventually it reaches an island or another monomer. In the first case it is incorporated into the island and becomes immobile; in the second case the two monomers form a new island, becoming immobile as well. The capture time $\tau_{c}$ can be related to the flux $F$ and the surface-density of monomers $\rho_{1}$ by

$$
\rho_{1} \sim F \tau_{c}
$$

For a description of a random walker it is more convenient to use the number of steps $n$ rather than time $t$. If the length of a time step $\Delta t$ and the lattice constant $\Delta x$ are given, the macroscopic diffusion constant $D$ is

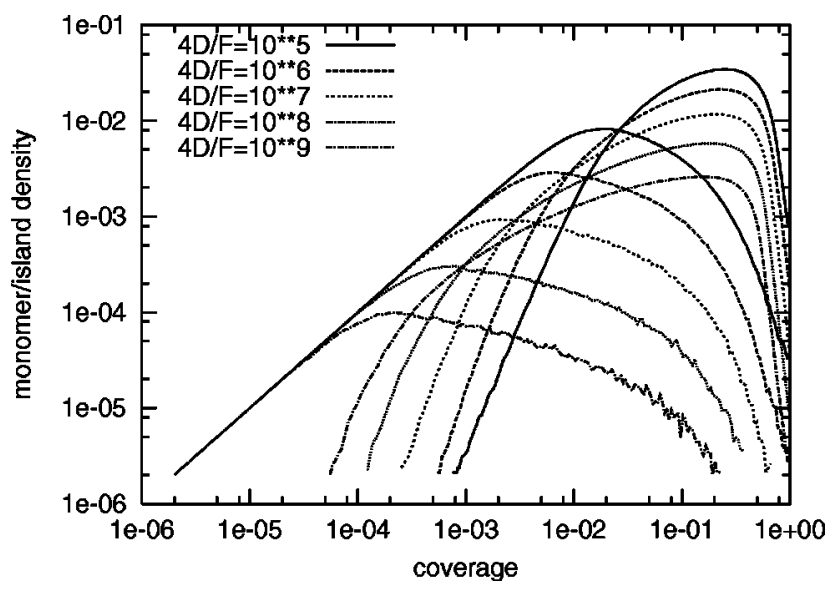

FIG. 2. Monomer $\rho_{1}$ and island densities $\rho$ vs coverage $\theta$ with elastic repulsion $U_{0} / T=4$, with $4 D / F$ ranging from $10^{5}$ to $10^{9}$.

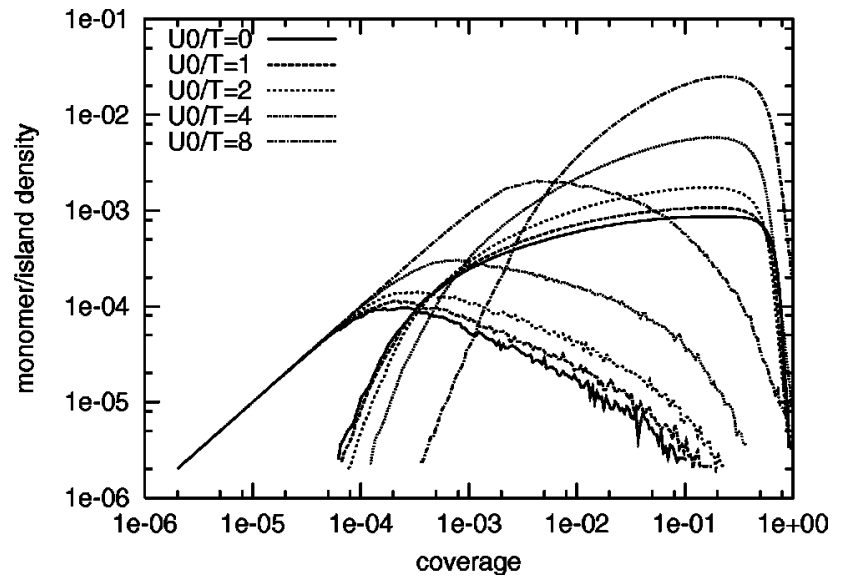

FIG. 3. Monomer $\rho_{1}$ and island densities $\rho$ vs coverage $\theta$ with $4 D / F=10^{8}$, with the interaction strength $U_{0} / T$ ranging from 0 to 8 .

$$
D \sim \frac{\Delta x^{2}}{4 \Delta t}
$$

The mean number of distinct sites $S(n)$ visited by a random walker on a simple two-dimensional square lattice after $n$ steps will be an important quantity. Because we will have to evaluate $S(n)$ for small $n$ due to our scaling arguments, we will have a closer look at this first passage time quantity. While earlier investigations [10-12] ended up using logarithmic corrections corresponding to the asymptotic expression $S(n) \sim \pi n / \ln n$, we see that for small $n \sim 10-1000$ the number of sites visited behaves more like $S(n) \sim \pi n / \ln 8 n$. An even better approximation is available by using the expansion derived by Henyey and Seshadri [9],

$$
S(n) \sim \frac{\pi n}{\ln 8 n} \sum_{j=0}^{\infty} \frac{c_{j}}{(\ln 8 n)^{j}}\left[1+O\left(\frac{1}{n}\right)\right],
$$

where the coefficients $c_{j}$ are given by derivatives of the $\Gamma$ function. Reference [9] contains explicit values tabulated up to $j=20$. Note that corrections to Eq. (3) are of order $O(1 / \ln n)$. We will consider these corrections by an additional term $1 /(a+b \ln n)$, with $a$ and $b$ originating from a numerical fit [9]. For numerical evaluation we will consider the first few terms of series (3) and the correction term. This approximation for $S(n)$ is reasonable for $n>1$. For the sake of simplicity, we will write $S(n)$ as

$$
S(n) \sim n f_{c}(n)
$$

where $f_{c}$ includes all deviations from linear behavior.

The reason why $S(n)$ is so important is because it can be related to the probability for a diffusing monomer to meet another adatom. Knowing the number of distinct lattice sites visited, we can interpret $\Delta x^{2} S\left(\tau_{c} / \Delta t\right)$ as the effective area covered by a monomer. The probability for a diffusing monomer of lifetime $\tau_{c}$ to collide with an island or another monomer is thus proportional to $\Delta x^{2} S\left(\tau_{c} / \Delta t\right) / \tau_{c}$, and the corresponding densities. 
At this point we introduce a set of rate equations, which considers the effects of monomer diffusion. The aim is to connect the density of diffusing monomers $\rho_{1}$ to the density of islands $\rho$, and to yield a description which is valid in the limit of low coverages $\theta$, where effects of island size and geometry can be ignored, and thus capture rates can be assumed to be independent of the island size. This is equivalent to the point island approximation, where islands are assumed to retain some infinitesimal lateral size during growth. The equations have the general forms

$$
\begin{gathered}
\frac{d \rho}{d t}=\frac{\Delta x^{2} S\left(\tau_{c} / \Delta t\right)}{\tau_{c}} \rho_{1}^{2}, \\
\frac{d \rho_{1}}{d t}=F-2 \frac{\Delta x^{2} S\left(\tau_{c} / \Delta t\right)}{\tau_{c}} \rho_{1}^{2}-\frac{\Delta x^{2} S\left(\tau_{c} / \Delta t\right)}{\tau_{c}} \rho \rho_{1} .
\end{gathered}
$$

The number of monomers $\rho_{1}$ [Eq. (6)] gains by deposition and loses by dimer formation as well as by the growth of existing islands, whereas the number of islands $\rho$ [Eq. (5)] increases by dimer formation only, because the growth of islands does not change their number.

With the coverage $\theta$ given by $\theta=F t$ and using Eqs. (4) and (1), one can define a characteristic length scale

$$
l_{c}\left(\rho_{1}\right)=\left[\frac{4 D}{F} f_{c}\left(\frac{4 D}{F} \frac{\rho_{1}}{\Delta x^{2}}\right)\right]^{1 / 4},
$$

and one has

$$
\begin{gathered}
\frac{d \rho}{d \theta}=l_{c}^{4}\left(\rho_{1}\right) \rho_{1}^{2}, \\
\frac{d \rho_{1}}{d \theta}=1-2 l_{c}^{2}\left(\rho_{1}\right) \rho_{1}^{2}-l_{c}^{4}\left(\rho_{1}\right) \rho \rho_{1} .
\end{gathered}
$$

Because the characteristic length scale $l_{c}$ shows a rather weak dependence on the coverage $\theta$, this dependence on $\theta$ can be ignored on the left hand sides of Eqs. (8) and (9). Scaled variables are now introduced as $\widetilde{\theta}=\theta l_{c}^{2}\left(\rho_{1}\right), \tilde{\rho}$ $=\rho l_{c}^{2}\left(\rho_{1}\right)$, and $\tilde{\rho}_{1}=\rho_{1} l_{c}^{2}\left(\rho_{1}\right)$. To be more explicit, the derivatives of scaled and unscaled variables are related by

$$
\frac{d \tilde{\rho}_{1}}{d \tilde{\theta}}=\frac{1+g(n)}{1+\frac{d \ln \rho_{1}}{d \ln \theta} g(n)} \frac{d \rho_{1}}{d \theta} \quad \text { with } \quad g(n)=\frac{n f_{c}^{\prime}(n)}{2 f_{c}(n)}
$$

and

$$
\frac{d \tilde{\rho}}{d \widetilde{\theta}}=\frac{1+\frac{d \ln \rho_{1}}{d \ln \rho} g(n)}{1+\frac{d \ln \rho_{1}}{d \ln \theta} g(n)} \frac{d \rho}{d \theta},
$$

where $g\left(n=\tau_{c} / \Delta t\right) \rightarrow 0$ with increasing lifetime $\tau_{c}$. Since $\rho_{1}$ can be locally approximated by $\rho_{1} \sim \theta^{\alpha}$, the term $d \ln \rho_{1} / d \ln \theta$ is just this local exponent $\alpha$, which in the

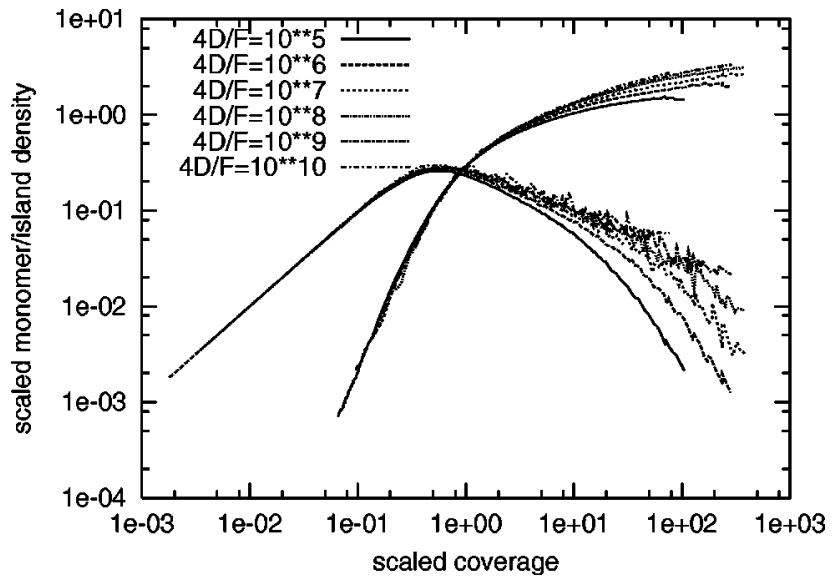

FIG. 4. Scaled monomer $\tilde{\rho}_{1}$ and island densities $\tilde{\rho}$ vs scaled coverage $\widetilde{\theta}$ without elastic repulsion $U_{0} / T=0$, with $4 D / F$ ranging from $10^{5}$ to $10^{10}$. The horizontal axis has been scaled with $l_{c}^{2}$ from Eq. (7).

present case can be assumed to be a number of $O(1)$. The term $d \ln \rho_{1} / d \ln \rho$ can be found to be of $O(1)$ by analogous arguments. Hence, in the limit of long lifetime $\tau_{c}$, the derivatives on the left hand sides of Eqs. (8) and (9) can be replaced as if the characteristic length $l_{c}$ did not depend on $\rho_{1}$.

By this scaling procedure the equations become dimensionless, and we obtain

$$
\begin{gathered}
\frac{d \tilde{\rho}}{d \widetilde{\theta}}=\tilde{\rho}_{1}^{2}, \\
\frac{d \tilde{\rho}_{1}}{d \widetilde{\theta}}=1-2 \tilde{\rho}_{1}^{2}-\tilde{\rho} \tilde{\rho}_{1},
\end{gathered}
$$

which allows for leading correction terms due to $f_{c}$ by the definition of the tilded variables.

While no exact solution can be found, we obtain the scaling behavior of the above equations. For sufficiently low coverages $\widetilde{\theta} \ll 1$ the loss terms in Eq. (13) are small compared with the flux term of order unity, and can be neglected. In this way $\tilde{\rho}_{1} \sim \widetilde{\theta}$, and thus $\tilde{\rho} \sim 1 / 3 \widetilde{\theta}^{3}$. At large coverages $\widetilde{\theta}$ the island density $\tilde{\rho}$ eventually becomes larger than the monomer density $\tilde{\rho}_{1}$, leading to a further decrease of $\tilde{\rho}_{1}$. With $\tilde{\rho}_{1} \ll \tilde{\rho}$ the middle term [Eq. (13)] can be ignored. Hence $\tilde{\rho}_{1} \tilde{\rho}$ has to be of the order of unity, which implies $\tilde{\rho}_{1} \sim \tilde{\rho}^{-1}$. Inserting into Eq. (12) yields $\tilde{\rho} \sim \widetilde{\theta}^{1 / 3}$ and $\tilde{\rho}_{1}$ $\sim \widetilde{\theta}^{-1 / 3}$. Because for large coverages the island size and geometric effects cannot be ignored, the rate equations fail to describe the high coverage regime, and one should regard the derived asymptotic behavior of $\tilde{\rho}_{1}$ and $\tilde{\rho}$ only as a qualitative result.

Figure 4 shows a scaled plot of the results shown in Fig. 1. Not surprisingly, the scaling prediction is fulfilled. The collapse is good up to $\widetilde{\theta} \sim 1$. The initial increase of $\tilde{\rho}_{1}$ and $\tilde{\rho}$ agrees with the predictions $\tilde{\rho}_{1} \sim \widetilde{\theta}$ and $\tilde{\rho} \sim 1 / 3 \widetilde{\theta}^{3}$. Note that 


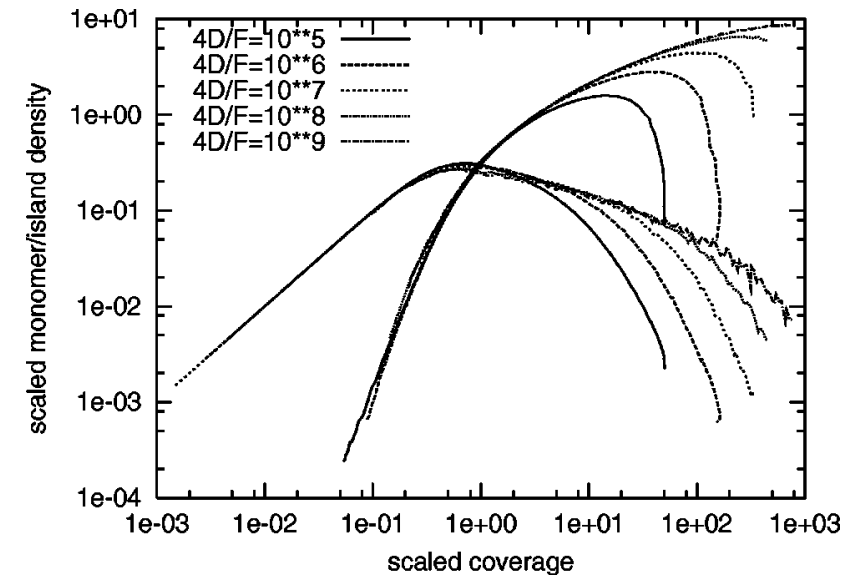

FIG. 5. Scaled monomer $\tilde{\rho}_{1}$ and island densities $\tilde{\rho}$ vs scaled coverage $\widetilde{\theta}$, with the elastic interaction strength fixed to $U_{0} / T=4$ and $4 D / F$ ranging from $10^{5}$ to $10^{9}$.

scaling by a constant $l_{c}$, i.e., setting $f_{c}(n)$ to an arbitrary constant, will not lead to coinciding peaks of the monomer densities; see the related plot in Ref. [12].

How can we incorporate the effects of elastic repulsion into the scheme of rate equations? We first note that the ratio $D / F$ is entirely the ratio of two time scales:

$$
D / F \sim \tau_{F} / \tau_{D}
$$

$\tau_{F}$ is the time it takes to deposit one monolayer, and $\tau_{D}$ is determined by the diffusion barrier $E_{D}$. The case of elastic repulsion gives rise to another time scale $\tau_{e}$, which is governed by the height of the elastic interaction potential $U_{0} / r^{3}$. Thus we have $\tau_{D} \sim \exp \left(E_{D} / T\right)$ and $\tau_{e} \sim \exp \left(U_{0} / T\right)$ governing the motion of the monomers. It is quite obvious that if $U_{0}$ $\ll E_{D}, \quad \tau_{D} \gg \tau_{e}$ or $U_{0} \gg E_{D}, \quad \tau_{D} \ll \tau_{e}$, the aggregation is triggered by the slower of the two processes. We argue that, in order to account for elastic repulsion, $\tau_{D}$ has to be replaced by $\tau_{D}+\tau_{e}$ in the denominator of Eq. (14), which is equivalent to the replacement

$$
\frac{4 D}{F} \rightarrow \frac{4 D}{F}\left[1+\exp \left(\frac{U_{0}-E_{D}}{T}\right)\right]^{-1} .
$$

As long as the interaction strength is lower than the diffusion barrier, elastic repulsion will only have a small effect in the nucleation regime, whereas with strong interaction the correction term to $D / F$ on the right hand side of Eq. (15) is of the order of $\exp \left(-U_{0} / T\right)$.

Including elastic interaction, the characteristic length scale $l_{c}$ changes to

$$
l_{c}=\left[\alpha f_{c}\left(\alpha \frac{\rho_{1}}{\Delta x^{2}}\right)\right]^{1 / 4},
$$

with

$$
\alpha=\frac{4 D}{F}\left[1+\exp \left(\frac{U_{0}-E_{D}}{T}\right)\right]^{-1} .
$$

Figure 5 shows scaled data from Fig. 2 with a fixed inter-

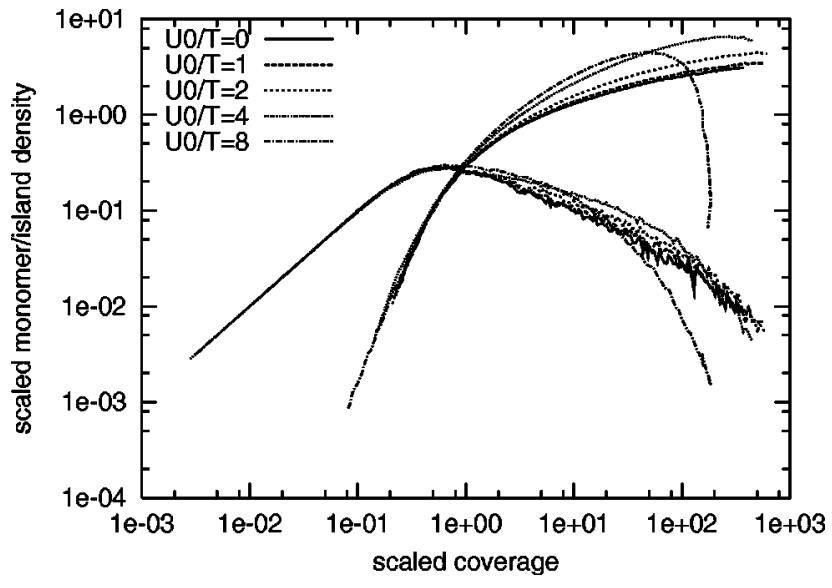

FIG. 6. Scaled monomer $\tilde{\rho}_{1}$ and island densities $\tilde{\rho}$ vs scaled coverage $\widetilde{\theta}$ with $4 D / F=10^{8}$ fixed, and interaction strength values $U_{0} / T=0,1,2,4$, and 8 .

action strength $U_{0} / T=4$, and $D / F$ varying over four orders of magnitude. The plot in Fig. 6 shows data from Fig. 3 scaled by $l_{c}$ according to Eq. (16). Here $4 D / F$ is fixed to $4 D / F=10^{8}$, and the interaction strength varies from $U_{0} / T$ $=0$ to $U_{0} / T=8$. In both cases the plotted data scale as predicted. Figure 7 shows scaled data of varying fluxes and interaction strength, merging all curves from Figs. 4, 5, and 6 into one graph.

As the scaling transformation only depends on $\alpha$, it has the forms

$$
\tilde{\rho}_{1}=\rho_{1} g_{\alpha}\left(\rho_{1}\right), \quad \tilde{\rho}=\rho g_{\alpha}\left(\rho_{1}\right), \quad \tilde{\theta}=\theta g_{\alpha}\left(\rho_{1}\right)
$$

with $g_{\alpha}$ depending on $\alpha$. Because the data collapse under the same mapping, the unscaled data have to be identical. All systems obeying the relation

$$
\frac{4 D}{F}=\alpha\left[1+\exp \left(\frac{U_{0}-E_{D}}{T}\right)\right],
$$

for fixed $\alpha$, show identical low temperature, low coverage nucleation properties. An example for the interpretation of

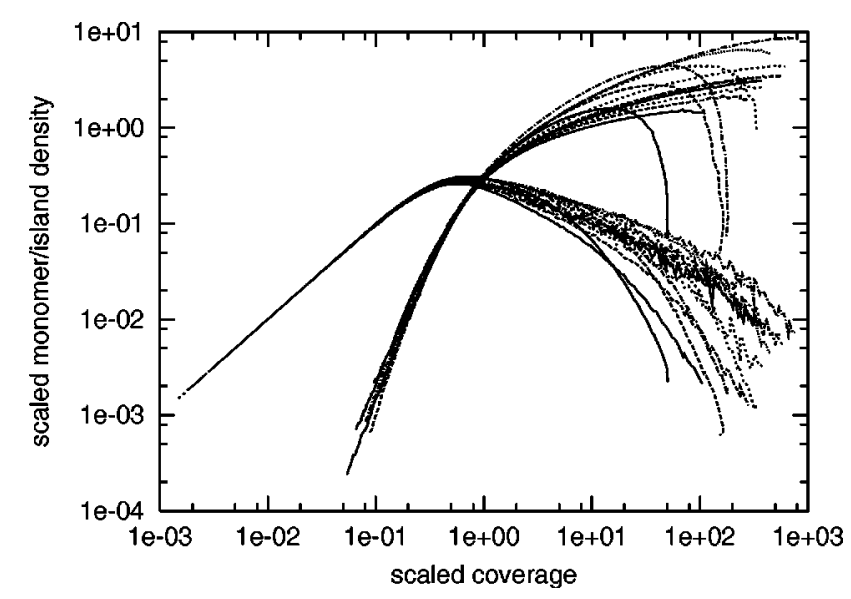

FIG. 7. Scaled monomer $\tilde{\rho}_{1}$ and island densities $\tilde{\rho}$ vs scaled coverage $\widetilde{\theta}$. All scaled curves are on one graph. 
these data is that the estimation of the diffusion constant $D$ from the usual scaling arguments [Eq. (7)] might fail in the presence of elastic effects; the estimate will turn out to be too small.

When the coverage $\theta$ increases, the island size and geometry will finally become important. The assumptions which led to Eqs. (12) and (13) are no longer valid. The probability for a diffusing monomer to attach to a specific island will be determined by the size and shape of this island. Because the attachment is irreversible, the islands shape will be fractal. If the islands are not too large, we expect the effective fractal dimension $d_{e f f}$ to increase continuously from the classical diffusion-limited aggregation value $\approx 1.7$ to the dimension of the lattice $d_{e f f}=2$, as the elastic interaction strength increases and the islands become more compact [8]. This should have an effect on diffusive aggregation, since a fractal island has a larger radius than a compact island of identical mass. At even higher coverages the deposition on top of an existing island cannot be neglected. Deposition on top of an island leads to an almost direct capture, since monomers diffuse to the edges. In the case of fractal islands the situation is similar, but monomers can be deposited between the inner branches of the fractal structure, an event leading to an almost direct capture as well. Because of the larger diameter, fractal growth should enhance this direct capture process. Nevertheless the influence of the fractal structure on the evolution of monomer and island densities has been shown to be rather weak in the absence of particle interaction [13]. However, this effect is noticeable, and makes it difficult to find scaling laws accounting for particle interaction in the higher coverage regimes. Furthermore, rate equations without spatial correlations usually have difficulties in predicting correct island size distributions even in the case where the mean island size turns out to be correct; see Ref. [13].

\section{CONCLUSION}

In summary, we have shown the effect of elastic interaction on low temperature submonolayer island formation in the low coverage regime by Monte Carlo simulation, and analyzed the results with scaling arguments. A particular result is that, with increasing elastic interaction strength $U_{0} / T$, the formation of islands is hampered, and island nucleation is deferred to higher values of coverage. In this respect the influence of an increased elastic interaction strength on the island nucleation process resembles the effect of an increased deposition flux $F$. A scaling relation [Eq. (16)] was found, connecting the strength of elastic interaction $U_{0}$, diffusion constant $D$, and flux $F$. For coverages that are not too large, universal scaling functions for cluster concentrations are obtained. This scaling regime holds as long as the average size of the clusters is much smaller than the distance between them. At larger coverages the mean cluster size becomes comparable to the distance between the clusters, and finally cluster coalescence has to be considered.

\section{ACKNOWLEDGMENTS}

We thank Y. Saito from KEIO University for valuable comments and suggestions. We furthermore appreciate support by a European travel grant "Procope."
[1] Y. Saito, Statistical Physics of Crystal Growth (World Scientific, Singapore, 1996).

[2] R. Nötzel, J. Temmyo, and T. Tamamura, Nature (London) 369, 131 (1994).

[3] R. Jullien, J. Kertesz, P. Meakin, and D. E. Wolf, Surface Disordering: Growth, Roughening and Phase Transitions (Nova, Commack, NY, 1993).

[4] J. Hardy and R. Bullough, Philos. Mag. 15, 237 (1967).

[5] K. H. Lau and W. Kohn, Surf. Sci. 65, 607 (1977).

[6] V. I. Marchenko and A. Y. Parshin, Zh. Éksp. Teor. Fiz. 79, 257 (1980) [Sov. Phys. JETP 52, 129 (1980)].
[7] A. F. Andreev and Y. A. Kosevich, Zh. Éksp. Teor. Fiz. 81, 1435 (1981) [Sov. Phys. JETP 54, 761 (1981)].

[8] J. Steinbrecher, H. Müller-Krumbhaar, E. Brener, C. Misbah, and P. Peyla, Phys. Rev. E 59, 5600 (1999).

[9] F. S. Henyey and V. Seshadri, J. Chem. Phys. 76, 5530 (1982).

[10] J. G. Amar, F. Family, and Pui-Man Lam, Phys. Rev. B 50, 8781 (1994).

[11] J. Villain, A. Pimpinelli, L. Thang, and D. Wolf, J. Phys. I 2, 2107 (1992).

[12] Lei-Han Thang, J. Phys. I 3, 935 (1993).

[13] G. S. Bales and D. C. Chrzan, Phys. Rev. B 50, 6057 (1994). 